\title{
Role of IL-I7 in plaque psoriasis: therapeutic potential of ixekizumab
}

\author{
This article was published in the following Dove Press journal: \\ Therapeutics and Clinical Risk Management \\ 13 March 2017 \\ Number of times this article has been viewed
}

\author{
Tessa L Hanley' \\ Zenas ZN Yiu ${ }^{2,3}$ \\ 'Fairfield General Hospital, Bury, \\ ${ }^{2}$ Centre for Dermatology, ${ }^{3}$ Centre \\ for Pharmacoepidemiology and Drug \\ Safety, Manchester Academic Health \\ Science Centre, Manchester, UK
}

\begin{abstract}
Developments in the understanding of the immunopathogenesis of psoriasis have identified interleukin (IL)-17 as the key proinflammatory cytokine in the pathogenesis of plaque psoriasis, with the consequent development of drugs that target this cytokine or associated receptors. Ixekizumab is a subcutaneously administered humanized monoclonal antibody, which acts to neutralize IL-17A. This article reviews the role of IL-17 in the pathogenesis of psoriasis, the biological and pharmacokinetics of ixekizumab and the safety profile and the clinical efficacy of ixekizumab in Phase III clinical trials. Phase III clinical trials of ixekizumab have so far demonstrated excellent early clinical efficacy, with a comparable safety profile to the existing biologic therapies for psoriasis. To further assess its position in the treatment algorithm for psoriasis, a further head to head RCT with secukinumab could be established, alongside comparative effectiveness studies from observational research. In addition, trials are needed to assess its role in those with tumor necrosis factor inhibitors/ustekinumab resistant disease. However, it is clear that the IL-17 antagonists have changed the benchmark for clinical efficacy, and it is likely that ixekizumab along with the other IL-17 antagonists are set to achieve a new standard of care in the treatment of moderate to severe plaque psoriasis.
\end{abstract}

Keywords: interleukin-17, psoriasis, IL-17, ixekizumab

\section{Introduction}

Psoriasis is a chronic, immune mediated, inflammatory skin disease, estimated to affect between $2 \%-3 \%$ of the UK population. ${ }^{1}$ Plaque psoriasis, the most common variant, is characterized by sharply demarcated, erythematous, pruritic plaques, with adherent silvery scales and accounts for around $90 \%$ of cases. ${ }^{2}$ The burden of the disease is not limited solely to the skin, with an estimated $30 \%$ patients developing psoriatic arthritis, which in its most aggressive form can cause permanent joint damage. ${ }^{3}$ Psoriasis is also associated with a number of other chronic conditions including cardiovascular disease, depression and metabolic syndrome. ${ }^{4}$ Given the significant morbidity and the psychosocial impact associated with the condition, it is of no surprise that it has severe consequences on patients' quality of life. ${ }^{5}$

Traditional therapies for the treatment of moderate to severe psoriasis include methotrexate, ciclopsorin, acitretin, and fumaric acid esters. ${ }^{6}$ However, they are limited by significant adverse effects and/or low effectiveness. A greater understanding of the pathogenesis of psoriasis has since allowed for the development of several highly effective biological therapies, revolutionizing the treatment of psoriasis and fulfilling the needs of this patient population. Current licensed biologics include the tumor necrosis factor (TNF)- $\alpha$ inhibitors etanercept, infliximab and adalimumab; interleukin(IL)-12/ IL-23 antagonist ustekinumab and the IL-17 antagonist secukinumab. ${ }^{6}$ The increased
Correspondence: Zenas ZN Yiu Dermatology Centre, Salford Royal NHS Foundation Trust, The University of Manchester, Manchester Academic Health Science Centre, Manchester, UK Tel +44 I6I 3060620

Email zenas.yiu@manchester.ac.uk hy
hereby accept the Terms. Non-commercial uses of the work are permitted without any further permission from Dove Medical Press Limited, provided the work is properly attributed. For permission for commercial use of this work, please see paragraphs 4.2 and 5 of our Terms (https://www.dovepress.com/terms.php). 
understanding of the significant role of IL-17 in the pathogenesis of psoriasis has led to the development of drugs targeting this cytokine, ${ }^{7}$ which include secukinumab, ixekizumab and brodalumab. Ixekizumab has subsequently been approved for use in patients with moderate to severe psoriasis by the US Food and Drug administration (FDA) and European Medicines Agency (EMA). Phase III clinical trials of Ixekizumab have so far shown significant and long lasting clinical response rates. ${ }^{8,9}$

\section{Methods}

The objective of this review was to explore the role of IL-17 in the pathogenesis of plaque psoriasis and to review the current evidence on the efficacy and safety of ixekizumab in the treatment of people with psoriasis. A literature review, undertaken by the authors, searched the PubMed database, for articles including the key words: "IL-17", "psoriasis", "ixekizumab" and "interleukin-17". Based on review of the abstracts, relevant articles, available in English, were selected for this review. The reference lists of relevant articles were reviewed for additional relevant literature.

\section{Role of IL-I 7 in the pathogenesis of psoriasis}

Although the exact pathogenesis of psoriasis yet to be elucidated, it is thought to be a complex interplay between environmental factors, T cells, dendritic cells, multiple cytokines and genetics, which dysregulate innate and adaptive immune responses in the skin. ${ }^{10,11}$ Over 40 susceptibility loci have been found to be associated with psoriasis using genomewide studies. ${ }^{12}$ These genes are involved in T cell signaling, antigen presentation and skin barrier function. ${ }^{13}$

The present model of psoriasis proposes that an unknown antigen or environmental trigger precipitates the activation of innate immune cells, including natural killer cells, plasmacytoid dendritic cells and macrophages. ${ }^{14,15}$ In a genetically susceptible individual, this leads to the activated cells producing the cytokines TNF- $\alpha$, IL- $1 \beta$ and IL- 6 . These cytokines in turn activate myeloid dendritic cells, acting as a bridge to innate and adaptive immune responses. The secretion of IL-12 and IL-23 by the myeloid dendritic cells causes naïve T cells to differentiate into helper type (Th)17 and type $1 \mathrm{Th}$ (Th1) cells. The maintenance and production of Th1 cells is primarily maintained by IL-12, whereas IL-23, composed of IL-23p19 and IL-23p40 protein subunits, is the predominant driver involved in Th17 cell differentiation. ${ }^{7,13-17}$ The effector $\mathrm{T}$ cells then migrate back into the skin where they release cytokines and chemokines driving the proliferation of keratinocytes. This is sustained by the keratinocytes, which amplify the inflammatory response by producing further cytokines, leading to a vicious cycle of inflammation. Mediators released by the effector T cells include interferon (IF) $\gamma$, IL-21 and TNF- $\alpha$ from Th1 cells and IL-17A, IL-17F and IL-22 from Th17 cells. Th17/IL-17 axis is now known to play an integral role in this inflammatory cascade, with IL-23 being pivotal for its stabilization. ${ }^{18}$

IL-17 cytokine is part of a family of cytokines, consisting of six ligand members (IL-17A to IL-17-F), with IL-17A as the prototypical ligand. Increased expression of IL-17A, IL-17C and IL-17F have been implicated in the pathogenesis of psoriasis. ${ }^{18}$ These interleukins interact with five transmembrane receptor subunits, IL-17 receptor A through IL-17 receptor E. ${ }^{13,17}$ IL-17A/IL-17A homodimers and IL-17A/ IL-17F heterodimers interact with heteromeric receptors consisting of two IL-17RA subunits and one IL-17RC subunit. IL-17A has multiple roles including neutrophil chemoattraction through upregulation of the expression of multiple neutrophil chemoattracting chemokines on keratinocytes, Th17 recruitment via expression of the keratinocyte chemokine CCL20, antimicrobial peptide expression - notably $\beta$ defensin 2 , stimulation of macrophages, endothelial cells and fibroblasts and potentiation of skin barrier dysfunction though downregulation of the expression of filaggrin in keratinocytes. ${ }^{10,11,13,17}$

This theory has been supported by the finding that Th17 cells are found in abundance in dermis of psoriatic lesion biopsies, with IL-17 mRNA expression of the key cytokines including IL-17A and IL-17F being shown to increase with disease activity, and normalize with treatment. ${ }^{19}$ Gene locus variants for IL-23 in genome wide association scans have also been associated with an increased risk of psoriasis. ${ }^{15}$ Clinical validation of the theory has been achieved though the success of targeted IL-17 antagonists in the treatment of plaque psoriasis. ${ }^{8,9}$

Currently there are three monoclonal antibodies targeting IL-17: secukinumab, ixekizumab and brodalumab. Ixekizumab and secukinumab both act to neutralize IL-7A; brodalumab targets the IL-17A receptor, thus inhibiting the IL-17 pathway. Secukinumab and ixekizumab have been approved by the FDA and EMA. Brodalumab is undergoing further clinical trials, and is yet to be licensed by either the FDA or EMA.

\section{Biological properties and pharmacokinetics of ixekizumab}

Ixekizumab (Eli Lilly) is a subcutaneously (SC)-administered humanized IgG4 monoclonal antibody, which selectively 
binds and neutralizes IL-17A..$^{20}$ The current recommended dosing regime is $160 \mathrm{mg}$ SC at week 0 , followed by $80 \mathrm{mg}$ at weeks 2, 4, 6, 8, 10 and 12, with maintenance dosing of $80 \mathrm{mg}$ every 4 weeks thereafter. ${ }^{21}$

Pharmacokinetic properties have been determined through intravenous (IV) and SC administration in cynomolgus monkeys. Following IV administration $1 \mathrm{mg} / \mathrm{kg}$, ixekizumab was eliminated with a mean halflife of 6.5 days compared to 10.3 days with the same dose administered SC, with an average maximal plasma concentration of $11.1 \mu \mathrm{g} / \mathrm{mL}$ at 72 hours post dose in the SC group, demonstrating high bioavailability from the $\mathrm{SC}$ site. ${ }^{22}$

In a Phase I, randomized, double blinded, placebocontrolled proof of concept study in rheumatoid arthritis, patients received either placebo or IV ixekizumab in a single dose of $0.06,0.2,0.6$ or $2 \mathrm{mg} / \mathrm{kg}$ followed by 8 weeks of evaluation (Part A) or IV ixekizumab $0.2,0.6$, or $2 \mathrm{mg} / \mathrm{kg}$ vs placebo every 2 weeks for five doses, with an evaluation period up to 16 weeks (Part B). The results showed the terminal elimination halflife to range from 15-18 days in part A of the study and 14-16 days in part B. ${ }^{23}$

More recently, an open label, Phase III study was undertaken (UNCOVER-A) to evaluate the effect of two drug delivery devices (prefilled syringe vs auto-injector) on the pharmacokinetics of ixekizumab. In this open label study, patients were randomized to receive ixekizumab $80 \mathrm{mg}$ every 2 weeks up until week 12 following a $160 \mathrm{mg}$ initial dose at week 0. Primary parameters were Cmax (the maximum concentration) and time to reach Cmax (tmax), the results being $\sim 15 \mu \mathrm{g} / \mathrm{mL}$ and $14.8 \mu \mathrm{g} / \mathrm{mL}$ for the prefilled syringe and the auto-injection devices, respectively, with both devices having a tmax of approximately 4 days. ${ }^{20,24}$

\section{Therapeutic potential of ixekizumab}

Three Phase III, multicenter, double-blinded, randomized controlled trials (RCTs) inclusive of 3,866 patients, UNCOVER-1, UNCOVER-2 and UNCOVER-3, have been published. ${ }^{8,9}$ Eligible patients were 18 years and older, had a diagnosis of chronic plaque psoriasis, at least $10 \%$ surface of their body surface area affected, a PASI score $>12$ (Psoriasis Area and Severity Index) and a static Physician's Global Assessment (sPGA) score $>3$ at screening and at baseline visits. ${ }^{8}$ Co-primary endpoints were: a reduction in PASI of at least $75 \%$ or sPGA scores of clear or minimal $(0 / 1)$ with at least a two point reduction from baseline at week 12 .

A total of 1,296 patients were assigned to the UNCOVER-1 trial, 1,224 patients to the UNCOVER-2 trial and 1,346 to the
UNCOVER-3 trial (Table 1). ${ }^{8,9}$ Patients were randomized to receive either SC injections of placebo, $80 \mathrm{mg}$ of ixekizumab every 2 weeks, or $80 \mathrm{mg}$ ixekizumab every 4 weeks. Both groups receiving ixekizumab received a starting dose of a $160 \mathrm{mg}$ of ixekizumab. Additional cohorts in the UNCOVER-2 and UNCOVER-3 trials were also randomized to receive $50 \mathrm{mg}$ of etanercept twice weekly.

In all three trials, primary endpoints were achieved with regards to the PASI 75 response and sPGA score 0/1 at week 12, with both ixekizumab dosing regimens showing greater efficacy compared to placebo (and etanercept in UNCOVER-2/3). At week 12 a PASI 75 response was achieved by $89.1 \%$ (UNCOVER-1), 89.7\% (UNCOVER-2) and $87.3 \%$ (UNCOVER-3) in those receiving ixekizumab every 2 weeks. This was significantly better than those demonstrated in the etanercept group at week $12(P<0.001)$, which achieved a PASI 75 response in $41.6 \%$ in UNCOVER-2 and $53.4 \%$ in UNCOVER-3 groups, respectively. At week 12 , the secondary endpoint of PASI 90 was achieved in 70.9\% (UNCOVER-1), 70.7\% (UNCOVER-2) and 68.1\% (UNCOVER-3) in the cohorts receiving ixekizumab every 2 weeks, compared to the etanercept cohort, in which a PASI 90 response was achieved in 18.7\% in UNCOVER-2 and $25.7 \%$ in UNCOVER-3 groups, respectively $(P<0.0001)$. As observed in Phase I and II clinical trials, clinical improvement was rapid, by week four approximately $50 \%$ of all patients in all studies had achieved a PASI 75\%. ${ }^{8,9}$

At week 12 in UNCOVER-3, patients entered a long-term extension arm receiving $80 \mathrm{mg}$ of ixekizumab every 4 weeks through to week 60. Patients in UNCOVER-1 and UNCOVER-2 trials that had responded to ixekizumab in the initial 12-week induction period were randomly reassigned to receive $80 \mathrm{mg}$ of ixekizumab every 4 weeks or every 8 weeks through to week 60. Patients in the UNCOVER-3 trial that initially received either ixekizumab either every 2 or every 4 weeks and went to receive $80 \mathrm{mg}$ every 4 weeks through weeks $12-60$, achieved a PASI 75 response in $83 \%$ and $80 \%$ of patients, respectively, at week 60. PASI 90 scores in the same groups at week 60 were $73 \%$ and $52 \%$ of patients, respectively $(P<0.001)^{8}$ (Table 2 ). In the pooled data from the UNCOVER-1 and UNCOVER-2 trials, patients that initially received ixekizumab every 2 weeks during the induction period, that went to receive ixekizumab either every 4 weeks, every 12 weeks, or placebo, attained PASI 75 responses of $83.3 \%, 49.1 \%$ and $9 \%$, respectively $(P<0.001)$. PASI 90 scores in the same groups at week 60 were $76.5 \%$, $40.6 \%$ and $4.7 \%$, respectively. ${ }^{8}$

The reports adhered well to the CONSORT checklist criteria. They were multicenter trials, with trial sites in over 





19 countries representing a varied treatment population. All three studies had $>99 \%$ power to detect superiority of each ixekizumab dosing regimen to placebo and $>93 \%$ to etanercept in the UNCOVER-2 and UNCOVER-3 trials for sPGA 0/1 and PASI 75 response rates. Randomization and allocation were achieved through computer-generated random sequence using an interactive voice response system (IVRS), with site personnel confirming they had located the correct assigned investigational product package by entering a confirmation number on the package on to the IVRS. Patients, investigators and study personnel were blinded to treatment allocation through to week 60 , with only a minimum number of sponsor personnel not in direct contact with study sites able to see the treatment assignments, thus limiting detection bias. Furthermore, all randomized patients were analyzed on an intention to treat basis to minimize the effect of attrition, with missing values for the PASI 75 or sPGA score being imputed conservatively as non-responders regardless of the reason for the missing data. However, patient withdrawals amongst the three trials at the week 12 primary end point were low $(<5 \%)$, thus limiting the effect of any attrition bias.

Weaknesses include lack of a long-term comparator arm for the extension phase after the initial 12-week induction period and a trial population of patients more likely to be biologic naive secondary to the exclusion of patients who had previously taken etanercept (UNCOVER-2 and UNCOVER-3 trials) compared to studies that have not excluded such patients. Additionally, the use of etanercept as a comparator group is poor given the alternatives available including adalimumab and ustekinumab which are the current gold standard treatments for psoriasis.

Subsequent subgroup analyses of the UNCOVER trials have also found ixekizumab to have a significant impact on itch severity and to be effective in the treatment of patients with scalp involvement, both of which have a major impact on patient quality of life. An analysis assessing the impact of itch severity in those treated with ixekizumab compared to etanercept and placebo in the UNCOVER-2 trial, found that by week 12 , changes to the numeric itch rating scores were (-5.2) and (-4.9) in the ixekizumab groups dosed every 2 weeks and every 4 weeks, respectively, compared to $(-3.6$ $P<0.001)$ in the etanercept cohort and $(-0.4 P<0.001)$ in the placebo cohort. ${ }^{25} \mathrm{~A}$ further subgroup analysis looking specifically at the effect of ixekizumab in scalp lesions of psoriasis sufferers in the UNCOVER-1, UNCOVER-2 and UNCOVER-3 trials found that in patients with baseline scalp involvement, nearly $75 \%$ achieved full clearance of their scalp lesions at 12 weeks when assessed using the Psoriasis Scalp Severity Index (PSSI) in those treated with ixekizumab $80 \mathrm{mg}$ every 2 weeks, after an initial starting dose of $160 \mathrm{mg}$. These outcomes were maintained though to week 60 when continuing treatment with $80 \mathrm{mg}$ of ixekizumab every 4 weeks. $^{26}$

A smaller, multicenter, open label, Phase III study, denoted UNCOVER-J evaluated the safety and clinical efficacy of ixekizumab in Japanese patients with moderate to severe psoriasis $(n=78)$, erythrodermic psoriasis $(n=8)$, and generalized pustular psoriasis $(n=5)$. Patients were given an initial ixekizumab dose of $160 \mathrm{mg}$, followed by $80 \mathrm{mg}$ every 2 weeks until 12. PASI 75 response rates were $83.3 \%, 100 \%$ and $80 \%$ in the moderate to severe psoriasis cohort, erythrodermic psoriasis cohort and generalized pustular psoriasis cohort, respectively. Efficacy was maintained through to 52 weeks in all cohorts. ${ }^{27}$ This study highlights the potential role of ixekizumab for erythrodermic psoriasis and generalized pustular psoriasis, but the study was limited by the lack of a control group and small sample size. ${ }^{27}$

Comparing the findings of the UNCOVER trials to the other IL-17 antagonists, ixekizumab achieved higher PASI 75 and PASI 90 response rates at week 12 and week 60 when dosed at $80 \mathrm{mg}$ every 2 weeks through to week 12 , followed by ixekizumab $80 \mathrm{mg}$ every 4 weeks through weeks $12-60$, following an initial $160 \mathrm{mg}$ starting dose. The FIXTURE trial, a Phase III, double blind, randomized control trial inclusive of 1,306 patients, found at week 12 PASI 75 responses of $77.1 \%$ and $67 \%$ in the $300 \mathrm{mg}$ and $150 \mathrm{mg}$ secukinumab groups, respectively (doses administered once weekly for 5 weeks and then every 4 weeks), ${ }^{28,29}$ compared with $44 \%$ and $4.9 \%$ in the twice weekly etanercept and placebo groups. Another Phase III trial, denoted as AMAGINE-1, a randomized, double blinded, placebo-controlled RCT inclusive of 661 patients comparing cohorts of brodalumab $210 \mathrm{mg}$ and $140 \mathrm{mg}$ to placebo, found the PASI 75 scores at week 12 to be $83.3 \%, 60.3 \%$ and $2.7 \%$, respectively. ${ }^{30}$ As yet there has been no head-to-head clinical trial performed between the IL-17 antagonists.

A multicenter randomized, double blinded, parallel group, Phase III study comparing ixekizumab to ustekinumab in patients which have previously failed treatment with conventional systemic agents, is currently underway and is due for completion in May 2017 (ClinicalTrials.gov ID NCT02561806). ${ }^{31}$ The preliminary results recently presented at the annual EADV (European Academy of Dermatology and Venereology) conference 2016 showed that at week 12 in those that received ixekizumab $80 \mathrm{mg}$ every 2 weeks 
until week 12, following an initial starting dose of $160 \mathrm{mg}$, achieved a PASI 90 response of $74 \%$, compared to $40 \%$ in the ustekinumab group dosed as per label. ${ }^{32}$ These results should be interpreted with caution until they are formally published, but highlight the potential superiority of ixekizumab to ustekinumab. Both secukinumab and brodalumab have also been shown to be superior to ustekinumab in separate Phase III RCTs. ${ }^{33,34}$

The efficacy of such drugs has led to the belief that the standard of care for patients with severe psoriasis should be elevated to PASI 90 instead of PASI 75 when assessing treatment response. Attaining such clinical responses is important because it has been demonstrated that increased clearance results in more significant improvements in health quality of life. ${ }^{35}$

\section{Psoriatic arthritis}

An important factor to consider with any biological therapy in treating psoriasis is its role in the treatment of psoriatic arthritis, as an estimated $30 \%$ of psoriasis sufferers will have coexistent psoriatic arthritis. ${ }^{3}$ The results of a Phase III double blinded, placebo-controlled and active comparatorcontrolled RCT denoted SPIRIT-P1 has recently been published. ${ }^{36}$ In this trial, biologic naïve patients with active psoriatic arthritis were randomized to receive adalimumab $40 \mathrm{mg}$ every 2 weeks, ixekizumab $80 \mathrm{mg}$ every 2 weeks, ixekizumab $80 \mathrm{mg}$ every 4 weeks or placebo. The primary objective was to assess superiority of ixekizumab over placebo as measured by those achieving an ACR (American College of Rheumatology) 20 response at week 24. An ACR 20 response was met at week 24 in $62.1 \%$ of patients receiving ixekizumab every 2 weeks and $57.9 \%$ in those receiving ixekizumab every 4 weeks, compared to $30.2 \%$ in the placebo group $P<0.001$. Adalimumab as the active reference achieved an ACR 20 response at week 24 in 57.4\% of patients compared with placebo $P<0.001 .{ }^{36}$ FUTURE 2, a double blind, Phase III RCT similarly showed promising results in psoriatic arthritis sufferers, with $54 \%$ of patients in the secukinumab $300 \mathrm{mg}$ cohort achieving an ACR 20 at week 24 , compared to $15 \%$ in the placebo group. ${ }^{37}$

\section{Safety and tolerability of ixekizumab}

Combined data from all three UNCOVER trials found that during the 12-week induction period, patients who received either dose of ixekizumab had higher rates of adverse events than those that were allocated to receive placebo. The percentage of patients having an adverse event were $58.4 \%$ and $58.5 \%$ in the ixekizumab groups dosed every 2 weeks and every 4 weeks, respectively, compared to $46.8 \%$ of patients in the placebo group. The most common adverse events occurring during this treatment period were nasopharyngitis, upper respiratory tract infections, injection site erythema and headache. ${ }^{8,9}$ In the 12-week induction period data, the exposure adjusted incidence rates because of at least once serious event were similar in those treated with either ixekizumab or placebo. The most common serious adverse event between weeks 0-12 in those receiving ixekizumab was infection in the form of cellulitis, which was found to occur in three patients. ${ }^{8,9}$

Looking specifically at infection as a serious adverse event, serious infections occurred in $1.4 \%$ of those with any ixekizumab exposure, equivalent to an incidence rate of 1.5 per 100 patient years. In comparison, the FIXTURE trial, an RCT comparing secukinumab to etanercept showed serious infection rates to be $1.5,0.6,0.3$ and 1.2 per 100 patient years for the $300 \mathrm{mg}, 150 \mathrm{mg}$, placebo and etanercept groups, respectively.

IL-17 is needed for immunity against candida albicans, with a rare genetic deficiency in humans being associated with recurrent candida infections. ${ }^{29}$ Therefore, it is not surprising that rates of oral and genital candidiasis (both confirmed and suspected cases) were found to be significantly higher in those receiving ixekizumab either every 2 weeks or every 4 weeks compared to placebo and etanercept. However, candidal infection did not meet the criteria for a serious adverse event in any of the trials and reassuringly there were no cases of invasive candidiasis. Furthermore, exposure adjusted incidence rates of candidal infections were noted to be similar during weeks $0-60$ than those found in weeks $0-12 .{ }^{8}$ Given this, it is important that patients on anti-IL-17 drugs are regularly monitored for skin and mucosal infections.

Another potential concern associated with ixekizumab use is neutropenia, as IL-17 has been shown to play an important role in neutrophil granulopoiesis. ${ }^{38}$ Results found the incidences of grade 1 and grade 2 neutropenia were noted to be more common amongst patients receiving ixekizumab, than those who received placebo during the 12-week induction period, with $7 \%$ of patients receiving ixekizumab every 2 weeks developing a grade 1 neutropenia compared to $2.9 \%$ in the placebo group. Only ten exposed patients $(0.2 \%)$ went on to develop a grade $3 / 4$ neutropenia, with none of these patients developing a serious infection as a result. ${ }^{8}$ Neutropenia has also been noted in trials with secukinumab and brodalumab. ${ }^{28,30}$ At present there is no published guidance to advise with regards to the frequency of monitoring for neutropenia.

Inflammatory bowel disease is an important comorbidity associated with psoriasis, the prevalence of which in Crohn's 
disease is estimated to be as high as $10 \% .{ }^{39}$ Early data from animal models suggested involvement of IL-17 in the pathogenesis of Crohn's, with its overexpression being demonstrated in the intestinal tissue in those with active Crohn's, ${ }^{40}$ however, a double blind, Phase II RCT found secukinumab to result in worsening of Crohn's disease, leading to early termination of the trial. ${ }^{41}$ In the pooled data from the three UNCOVER trials through weeks $0-60$, seven patients developed ulcerative colitis and four developed Crohn's disease in those receiving ixekizumab, with three additional patients developing Crohn's following the randomized withdrawal period whilst receiving placebo. This has led to guidance recommending cautious use in those with inflammatory bowel disease. ${ }^{21}$

A further potential concern for safety of IL-17 antagonists was highlighted by the withdrawal of Amgen from the development of brodalumab, following incidences of suicidal ideation and one successful attempt at suicide during the 52-week trial period within AMAGINE-2. Another patient in the same trial completed suicide after week 52 in the open label extension study. ${ }^{42}$ All patients in the UNCOVER trials were screened for depressive illness at outset using questionnaires, with those with any history of prior suicide attempts, an uncontrolled neuropsychiatric disorder or frequent active suicidal ideation being excluded from the trial. Nine of 3,736 patients exposed to ixekizumab between weeks 0-60 were reported to have suffered a serious adverse event in the form of a psychiatric disorder. ${ }^{8}$ Of these, two were suicide attempts in the UNCOVER-2 and UNCOVER-3 trials. No cases of completed suicide occurred in any of the UNCOVER trials. The FIXTURE and ERASURE trials reported no suicide attempts with patients on secukinumab. ${ }^{28}$ Given these findings, close monitoring will be required to ascertain the real risk of treatment emergent depression and suicide. This will be best identified through prospective registry data given its relatively rare occurrence.

Another important factor which needs to be considered when commencing any biologic is its immunogenicity. ${ }^{43}$ Anti-drug antibodies detected by an affinity capture elution (ACE) immunogenicity assay developed in 103 patients (9\%) is receiving ixekizumab every 2 weeks during the induction period in the three UNCOVER trials. Nineteen of these patients had had titers of antibodies $(>1 ; 1,280)$ which was accompanied by a reduced clinical response compared to those patients with low to moderate titers of antibodies. ${ }^{8}$ Those patients receiving ixekizumab every 2 weeks who did not have high titers of antibodies and had a response at week 12 were randomly assigned to receive ixekizumab $80 \mathrm{mg}$ every 4 weeks through weeks $12-60$. Clinical responses in this group were found to be maintained with no significant differences between the patients with low or moderate titres. ${ }^{8}$

Further considerations for patient tolerability include frequency of injections. The IL-17 inhibitors have relatively frequent dosing regimens; ixekizumab (2/4 weekly injections) and secukinumab (4 weekly injections), compared to ustekinumab (12 weekly), which could potentially pose issues with patient adherence. Furthermore, the intensive initial dosing regimen of ixekizumab every 2 weeks may have an impact on the early adverse event profile of the drug, which should be assessed in real-world data through prospective registries.

\section{Conclusion}

Phase III clinical trials of ixekizumab have so far demonstrated excellent clinical efficacy and an acceptable safety profile. Additionally, ixekizumab has demonstrated high clinical efficacy for the treatment psoriatic arthritis comparable to the anti TNF inhibitors, the current gold standard in the treatment of psoriatic arthritis. These findings may secure it a place as a first-line treatment in those with moderate to severe psoriasis alongside secukinumab, adalimumab, and ustekinumab. However, to further assess its position in the treatment algorithm a head to head study with secukinumab should be established, alongside comparative effectiveness studies from observational research, with additional trials needed to assess its role in those with TNF inhibitors/ ustekinumab resistant disease. Data on safety and persistence of ixekizumab and the other IL-17 antagonists will need to be ascertained through post marketing surveillance in biologics registers, eg, The British Association of Dermatologists Biologic Interventions Register.

However, it is clear that the IL-17 antagonists have changed the benchmark for clinical efficacy, and it is likely that ixekizumab along with the other IL-17 antagonists are set to achieve a new standard of care in the treatment of moderate to severe plaque psoriasis, with a PASI 90 response, if not PASI 100 being the new gold standard. The addition of ixekizumab will provide a valuable treatment option to patients suffering from severe psoriasis.

\section{Disclosure}

The authors report no conflicts of interest in this work.

\section{References}

1. Springate DA, Parisi R, Kontopantelis E, Reeves D, Griffiths CE, Ashcroft DM. Incidence, prevalence and mortality of patients with psoriasis: a UK population-based cohort study. Br J Dermatol. 2016. [Epub ahead of print] 
2. Griffiths CEM, Barker JN. Pathogenesis and clinical features of psoriasis. Lancet. 2007;370(9583):263-271.

3. Zachariae H. Prevalence of joint disease in patients with psoriasis: implications for therapy. Am J Clin Dermatol. 2003;4(7):441-447.

4. Ni C, Chiu MW. Psoriasis and comorbidities: links and risks. Clin Cosmet Investig Dermatol. 2014;7:119-132.

5. Rapp SR, Feldman SR, Exum ML, Fleischer AB Jr, Reboussin DM. Psoriasis causes as much disability as other major medical diseases. J Am Acad Dermatol. 1999;41(3):401-407.

6. National Clinical Guideline C. National Institute for Health and Clinical Excellence: Guidance 2012. Psoriasis: Assessment and Management of Psoriasis. London: Royal College of Physicians (UK).

7. Jinna S, Strober B. Anti-interleukin-17 treatment of psoriasis. J Dermatolog Treat. 2016;27(4):311-315.

8. Gordon KB, Blauvelt A, Papp KA, et al. Phase 3 trials of ixekizumab in moderate-to-severe plaque psoriasis. $N$ Engl J Med. 2016;375(4): $345-356$.

9. Griffiths CE, Reich K, Lebwohl M, et al. Comparison of ixekizumab with etanercept or placebo in moderate-to-severe psoriasis (UNCOVER-2 and UNCOVER-3): Results from two Phase 3 randomised trials. Lancet. 2015;386(9993):541-551

10. Campa M, Mansouri B, Warren R, Menter A. A review of biologic therapies targeting IL-23 and IL-17 for use in moderate-to-severe plaque psoriasis. Dermatol Ther (Heidelb). 2016;6(1):1-12.

11. Girolomoni G, Mrowietz U, Paul C. Psoriasis: rationale for targeting interleukin-17. Br J Dermatol. 2012;167(4):717-724.

12. Mahil SK, Capon F, Barker JN. Genetics of psoriasis. Dermatol Clin. 2015;33(1):1-11.

13. Lonnberg AS, Zachariae C, Skov L. Targeting of interleukin-17 in the treatment of psoriasis. Clin Cosmet Investig Dermatol. 2014;7: 251-259.

14. Gaspari AA, Tyring S. New and emerging biologic therapies for moderateto-severe plaque psoriasis: Mechanistic rationales and recent clinical data for IL-17 and IL-23 inhibitors. Dermatol Ther. 2015;28(4):179-193.

15. Nwe SM, Champlain AH, Gordon KB. Rationale and early clinical data on IL-17 blockade in psoriasis. Expert Rev Clin Immunol. 2013;9(7): 677-682.

16. Ariza ME, Williams MV, Wong HK. Targeting IL-17 in psoriasis: from cutaneous immunobiology to clinical application. Clin Immunol. 2013;146(2):131-139.

17. Martin DA, Towne JE, Kricorian G, et al. The emerging role of IL-17 in the pathogenesis of psoriasis: preclinical and clinical findings. J Invest Dermatol. 2013;133(1):17-26.

18. Gooderham M, Posso-De Los Rios CJ, Rubio-Gomez GA, Papp K. Interleukin-17 (IL-17) inhibitors in the treatment of plaque psoriasis: a review. Skin Therapy Lett. 2015;20(1):1-5.

19. Lowes MA, Kikuchi T, Fuentes-Duculan J, et al. Psoriasis vulgaris lesions contain discrete populations of Th1 and Th17 T cells. J Invest Dermatol. 2008;128(5):1207-1211.

20. Vu HTT, Gooderham M, Papp K. Ixekizumab for treatment of adults with moderate-to-severe plaque psoriasis and psoriatic arthritis. Expert Rev Clin Pharmacol. 2016;9(11):1423-1433.

21. Summary of product characteristics. Taltz $80 \mathrm{mg}$ solution for injection in pre-filled syringe. Available at: http://www.ema.europa.eu/docs/en_GB/ document_library/EPAR_-_Product_Information/human/003943/ WC500205804.pdf. Accessed 26/10/2016.

22. Liu L, Lu J, Allan BW, et al. Generation and characterization of ixekizumab, a humanized monoclonal antibody that neutralizes interleukin17A. J Inflamm Res. 2016;9:39-50.

23. Genovese MC, Van den Bosch F, Roberson SA, et al. LY2439821, a humanized anti-interleukin-17 monoclonal antibody, in the treatment of patients with rheumatoid arthritis: A Phase I randomized, double-blind, placebo-controlled, proof-of-concept study. Arthritis Rheum. 2010;62(4):929-939.

24. Callis Duffin K, Bagel J, Bukhalo M, et al. Phase 3, open-label, randomized study of the pharmacokinetics, efficacy and safety of ixekizumab following subcutaneous administration using a prefilled syringe or an autoinjector in patients with moderate-to-severe plaque psoriasis (UNCOVER-A). J Eur Acad Dermatol Venereol. 2016;31(1):107-113.
25. Kimball AB, Nikai E, Zhu B, Carlier H, Yosipovitch G. Ixekizumab impact on itch severity compared to etanercept and placebo: Results from UNCOVER-2, a Phase 3 trial in patients with moderate-to-severe plaque psoriasis. Journal of Investigative Dermatology. 2015;135:S19.

26. Reich K, Leonardi C, Lebwohl M, et al. Sustained response with ixekizumab treatment of moderate-to-severe psoriasis with scalp involvement: results from three Phase 3 trials (UNCOVER-1, UNCOVER-2, UNCOVER-3). J Dermtol Treat. 2016:1-6.

27. Saeki H, Nakagawa H, Ishii T, et al. Efficacy and safety of open-label ixekizumab treatment in Japanese patients with moderate-to-severe plaque psoriasis, erythrodermic psoriasis and generalized pustular psoriasis. J EurAcad Dermatol Venereol. 2015;29(6):1148-1155.

28. Langley RG, Elewski BE, Lebwohl M, et al. Secukinumab in plaque psoriasis - results of two Phase 3 trials. $N$ Engl J Med. 2014;371(4): 326-338.

29. Rothstein B, Gottlieb A. Secukinumab for treating plaque psoriasis. Expert Opin Biol Ther. 2016;16(1):119-128.

30. Papp KA, Reich K, Paul C, et al. A prospective Phase III, randomized, double-blind, placebo-controlled study of brodalumab in patients with moderate-to-severe plaque psoriasis. Br J Dermatol. 2016;175(2):273-286.

31. Eli Lilly and Company. A Study of Ixekizumab (LY2439821) in Participants With Moderate-to-Severe Plaque Psoriasis (IXORA-S). Available from https://clinicaltrials.gov/ct2/show/NCT02561806?ter $\mathrm{m}=\mathrm{ixekizumab \& rank=12.} \mathrm{Accessed} \mathrm{October} \mathrm{26,} 2016$.

32. 25th EADV Congress. Late-breaking insight: Ixekizumab vs ustekinumab head-to-head data in psoriasis. [Video on the Internet]. October 4, 2016. https://www.youtube.com/watch?v=pTasylB3yAU, 2016. Accessed January 27, 2017.

33. Blauvelt A, Reich K, Tsai TF, et al. Secukinumab is superior to ustekinumab in clearing skin of subjects with moderate-to-severe plaque psoriasis up to 1 year: Results from the CLEAR study. $J$ Am Acad Dermatol. 2016;76(1):60-69.

34. Lebwohl M, Strober B, Menter A, et al. Phase 3 Studies Comparing Brodalumab with Ustekinumab in Psoriasis. NEngl J Med. 2015;373(14): 1318-1328.

35. Takeshita J, Callis Duffin K, Shin DB, et al. Patient-reported outcomes for psoriasis patients with clear versus almost clear skin in the clinical setting. J Am Acad Dermatol. 2014;71(4):633-641.

36. Mease PJ, van der Heijde D, Ritchlin CT, et al. Ixekizumab, an interleukin-17A specific monoclonal antibody, for the treatment of biologic-naive patients with active psoriatic arthritis: results from the 24-week randomised, double-blind, placebo-controlled and active (adalimumab)-controlled period of the Phase III trial SPIRIT-P1. Ann Rheum Dis. 2016.

37. McInnes IB, Mease PJ, Kirkham B, et al. Secukinumab, a human anti-interleukin-17A monoclonal antibody, in patients with psoriatic arthritis (FUTURE 2): a randomised, double-blind, placebo-controlled, Phase 3 trial. Lancet. 2015;386(9999):1137-1146.

38. Forlow SB, Schurr JR, Kolls JK, Bagby GJ, Schwarzenberger PO, Ley K. Increased granulopoiesis through interleukin-17 and granulocyte colony-stimulating factor in leukocyte adhesion molecule-deficient mice. Blood. 2001;98(12):3309-3314.

39. Yiu ZZN, Griffiths CEM. Interleukin 17-A inhibition in the treatment of psoriasis. Expert Rev Clin Immunol. 2016;12(1):1-4.

40. Fitzpatrick LR. Inhibition of IL-17 as a pharmacological approach for IBD. In Rev Immunol. 2013;32(5-6):544-555.

41. Hueber W, Sands BE, Lewitzky S, et al. Secukinumab, a human antiIL-17A monoclonal antibody, for moderate to severe Crohn's disease: unexpected results of a randomised, double-blind placebo-controlled trial. Gut. 2012;61(12):1693-1700.

42. Farahnik B, Beroukhim K, Abrouk M, et al. Brodalumab for the Treatment of Psoriasis: A Review of Phase III Trials. Dermatol Ther (Heidelb). 2016;6(2):111-124.

43. Warren RB, Smith CH, Yiu ZZ, et al. Differential Drug Survival of Biologic Therapies for the Treatment of Psoriasis: A Prospective Observational Cohort Study from the British Association of Dermatologists Biologic Interventions Register (BADBIR). J Invest Dermatol. 2015; 135(11):2632-2640. 
Therapeutics and Clinical Risk Management

Dovepress

\section{Publish your work in this journal}

Therapeutics and Clinical Risk Management is an international, peerreviewed journal of clinical therapeutics and risk management, focusing on concise rapid reporting of clinical studies in all therapeutic areas, outcomes, safety, and programs for the effective, safe, and sustained use of medicines. This journal is indexed on PubMed Central, CAS,

EMBase, Scopus and the Elsevier Bibliographic databases. The manuscript management system is completely online and includes a very quick and fair peer-review system, which is all easy to use. Visit http://www.dovepress.com/testimonials.php to read real quotes from published authors.

Submit your manuscript here: http://www.dovepress.com/therapeutics-and-clinical-risk-management-journal 